\title{
Vitreous Noise on Optical Coherence Tomography as an Early Finding of Brolucizumab-Related Intraocular Inflammation
}

\author{
Yuji Yoshikawa Tomoyuki Kumagai Kei Shinoda \\ Department of Ophthalmology, Faculty of Medicine, Saitama Medical University, Saitama, \\ Japan
}

\section{Keywords}

Vitreous haze $\cdot$ Brolucizumab $\cdot$ Age-related macular degeneration · Intraocular inflammation

\begin{abstract}
We describe a case of brolucizumab-related intraocular inflammation (IOI) detected using vitreous haze on optical coherence tomography (OCT) at an early stage before the patient was aware of any symptom. A 69-year-old female presented with decreased right vision. The patient was diagnosed with pachychoroidal neovasculopathy and started intravitreal aflibercept (IVA) with a $3+$ treat-and-extend strategy (TAE). Although the serous retinal detachment (SRD) disappeared after IVA treatment, the patient was managed with treatment every 4 weeks without extending the treatment interval To shorten the treatment interval, intravitreal brolucizumab (IVBr) was started 44 weeks after starting IVA treatment. After initiating IVBr treatment, the SRD completely disappeared. However, 16 weeks after starting IVBr, OCT showed noise in the vitreous cavity, which had not been seen before, and infrared images showed a black smoke-like shadow over the macula. Despite these findings, the patient had no subjective symptoms, and so IVBr was readministered with an 8-week TAE interval. Five days after IVBr treatment, vitreous inflammatory cells were observed, and the noise in the vitreous cavity and the smoke-like shadow in the infrared image were further enhanced. We diagnosed the patient with brolucizumab-related IOI, and anti-inflammatory treatment was initiated. After extensive treatment, the vitreous opacity gradually disappeared, and the vitreous noise on OCT and the black smoke-like shadow on infrared images disappeared. IOI may have already been present 16 weeks after starting IVBr treatment, when we judged that there was no inflammation and IVBr was re-administered. When following patients receiving $\mathrm{IVBr}, \mathrm{IOI}$ may be detected by OCT at an earlier stage by evaluating vitreous haze.


Yoshikawa et al.: Vitreous Noise on OCT and Brolucizimab-Related IOI

\section{Introduction}

Age-related macular degeneration is a chronic progressive disease that causes irreversible visual impairment. The main modern anti-vascular endothelial growth factors (antiVEGFs) are ranibizumab [1] and aflibercept [2,3]. While strict treatment can maintain visual function, the SEVEN-UP study reported that the improvement in visual acuity reported in the MARINA/ANCHOR study could not be maintained at 7 years [4]. In the real world, it is difficult to maintain strict treatment, and long-term maintenance of vision is reported to be difficult [5]. The EVEREST II study reported that the number of intravitreal ranibizumab injections for polypoidal choroidal vasculopathy could be reduced by combining initial photodynamic therapy [6] to become the mainstay of current polypoidal choroidal vasculopathy treatment. Recently, brolucizumab has been introduced as a new anti-VEGF drug that can extend the treatment interval [7], and the HAWK and HARRIER trials showed that fewer injections are required and that visual outcomes are noninferior to aflibercept [8]. In contrast, brolucizumab was reported to cause intraocular inflammation (IOI) in $4.6 \%$ of patients after injection and sometimes causes occlusive vasculitis, resulting in irreversible visual impairment [9]. IOI, including occlusive vasculitis, is considered to be a pathological condition of the same spectrum, and the importance of early detection and treatment has been asserted [10]. We report a case of IOI that could be detected early using optical coherence tomography (OCT).

\section{Case Report}

A 69-year-old female visited our clinic with decreased vision in her right eye. The bestcorrected visual acuity (BCVA) was 20/25, and intraocular pressure was $17 \mathrm{~mm} \mathrm{Hg}$. Slit-lamp examination revealed serous retinal detachment (SRD) in the macula, and OCT (Spectralis, OCT2 Module; Heidelberg Engineering GmbH, Heidelberg, Germany) showed SRD, irregular retinal pigment epithelium, and pachyvessels. Fluorescein fundus angiography showed hyperfluorescent lesions in the early to late stage, indocyanine fundus angiography showed localized choroidal hyperpermeability in the late stage, and OCT angiography (OCTA) showed flow signal on B-scan images coincident with the site of retinal pigment epithelium irregularity (Fig. 1). Thus, the patient was diagnosed with pachychoroidal neovasculopathy and started intravitreal aflibercept (IVA) using a 3+ treat-and-extend strategy (TAE). Although SRD disappeared at 8 weeks after IVA treatment, SRD recurrence was observed at 16 weeks after starting IVA treatment, and the patient was managed with treatment every 4 weeks without extending the treatment interval. The BCVA was maintained at 20/22, but the SRD did not completely disappear, and intravitreal brolucizumab (IVBr) was started 44 weeks after starting IVA treatment (Fig. 2). After initiating IVBr treatment, the SRD completely disappeared. At the 16-week examination after the start of IVBr, her visual acuity was unchanged at 20/22, and there were no inflammatory findings, such as anterior chamber cells, vitreous opacity, or vasculitis, in the slit-lamp examination and fundus photograph. However, OCT showed noise in the vitreous cavity, which had not been seen before, and infrared images showed a black smoke-like area over the macula (Fig. 2, 3). Moreover, OCT showed that the luminance of the retinal image underlying the vitreous noise decreases, corresponding to the darkest smoke-like shadow area (online suppl. Figure; for all online suppl. material, see www.karger.com/doi/10.1159/000518809). This is deemed to stem from the weakness of the reflected laser signal by the scattering effect of the vitreous haziness, which we refer to as "shadow." Despite these findings, the patient had no subjective symptoms, and so the attending doctor decided to re-administer IVBr at 16 weeks at with 8-week TAE interval. Five days after IVBr, her visual acuity had decreased to 20/100. Slit-lamp

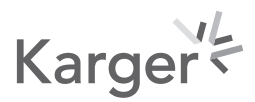




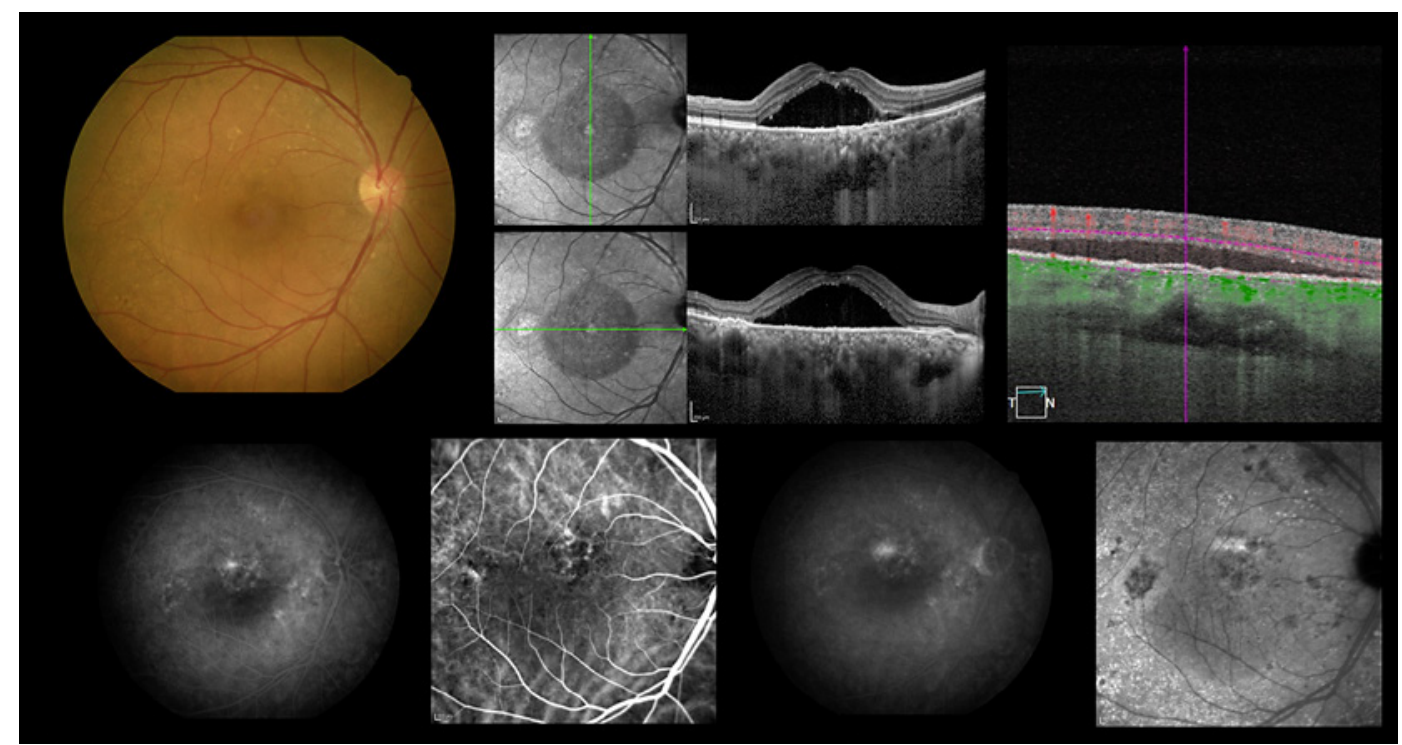

Fig. 1. Fundus photograph shows serous retinal detachment in the macula (upper left). Optical coherence tomography showed serous retinal detachment and pachyvessels (upper middle), and optical coherence tomography angiography showed retinal pigmented epithelium irregularity and slight flow signal (upper right). Fluorescein and indocyanine angiography examination showed a hyperfluorescent lesion in the upper macula, suggesting the presence of choroidal neovascularization (lower row).

examination revealed anterior chamber and vitreous inflammatory cells, and fundus photograph and fundus autofluorescence showed vitreous opacities. The vitreous noise in the OCT and the black smoke-like shadow in the infrared image were further enhanced. Fluorescein fundus angiography examination showed retinal vasculitis, which was diagnosed as brolucizumab-related IOI. As such, betamethasone drops were administered 6 times a day, $40 \mathrm{mg}$ subtenon triamcinolone acetonide was injected in the right eye, and $40 \mathrm{mg}$ oral prednisolone was administered per day. After extensive treatment, the vitreous opacity gradually disappeared on fundus photograph, and the noise in the vitreous cavity on OCT and the opacity on the infrared images disappeared. The BCVA was 20/22 at 24 weeks after starting IVBr treatment (Fig. 3).

\section{Discussion/Conclusion}

This case shows that it might be possible to detect vitreous inflammatory conditions using OCT at a time when IOI could not be detected based on the patient's subjective findings and fundus examination. OCT examination is known to be susceptible to various artifacts [11]. However, speckle noise can be removed by the averaging method [12]. A previous study reported that vitreous opacity can be objectively evaluated by OCT, and vitreous cells and vitreous haze can be observed depending on the degree of uveitis $[13,14]$. In our case, infrared images at 4 and 8 weeks after the start of IVBr treatment showed a suspicious shadow or dark area, and the vertical scan OCT images showed some vitreous haze and vitreous noise. However, vitreous haze is seen over the fovea where there is no shadow on the infrared image and no vitreous noise on the horizontal scan image. Furthermore, there is no change in the luminance of the retinal image underlying the noise. Therefore, we could not conclude that the shadow seen on the infrared image is vitreous noise suggestive of vitreous inflammation. 


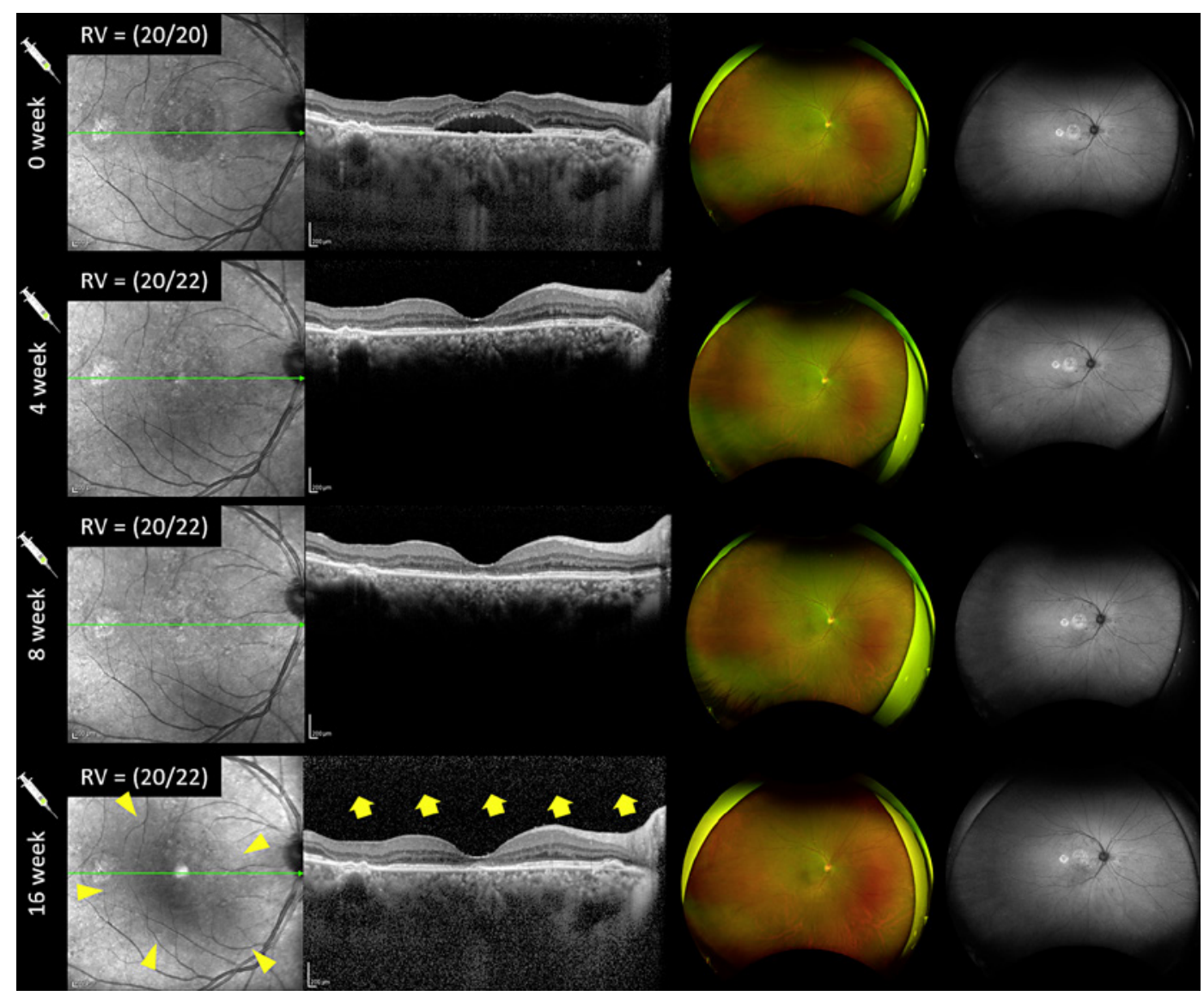

Fig. 2. Refractory SRD in response to intravitreal aflibercept was treated by switching to IVBr injection $(0,4$, and 8 weeks). SRD disappeared, and fundus photograph and fundus autofluorescence showed no obvious inflammatory findings. Sixteen weeks later, infrared images showed black smoke-like shadows (yellow arrowhead), and optical coherence tomography showed vitreous noise that had not been seen before (yellow arrow). However, there was no decrease in visual acuity or subjective symptoms, and there were no obvious inflammatory findings on fundus photograph or autofluorescence tests, so IVBr injection was administered as the first IVBr injection of a treat-and-extend regimen. SRD, serous retinal detachment; IVBr, intravitreal brolucizumab.

However, the infrared image showed an obvious black smoke-like shadow, and the OCT image at 16 weeks shows obvious vitreous noise in both the horizontal and vertical scans. In the area where the darkest shadow is seen in the infrared image, the luminance of the retinal image underlying the vitreous noise decreases, indicating that there is something in the vitreous that interferes with the OCT scan (online suppl. Figure). Five days after re-administration of IVBr (17 weeks after IVBr initiation), obvious IOI was present. After steroid treatment, the vitreous cells and vitreous haze, which were seen in the OCT image, as well as the black smoke-like shadow that was seen in the infrared image, gradually disappeared. At 24 weeks after starting IVBr, the vitreous cells completely disappeared and the vitreous haze almost disappeared, and then OCT images became noise free as before 16 weeks. This suggests that the black smoke-like shadow on the infrared images and the vitreous noise on OCT images at 16 weeks after the start of IVBr injection were likely vitreous haze reflecting IOI, and IOI was already present at 16 weeks after the start of IVBr treatment, when we judged that there was no inflammation and when IVBr was re-administered. We cannot deny the possibility that silent inflammation was already present at 4 or 8 weeks after the IVBr injection, and that the 


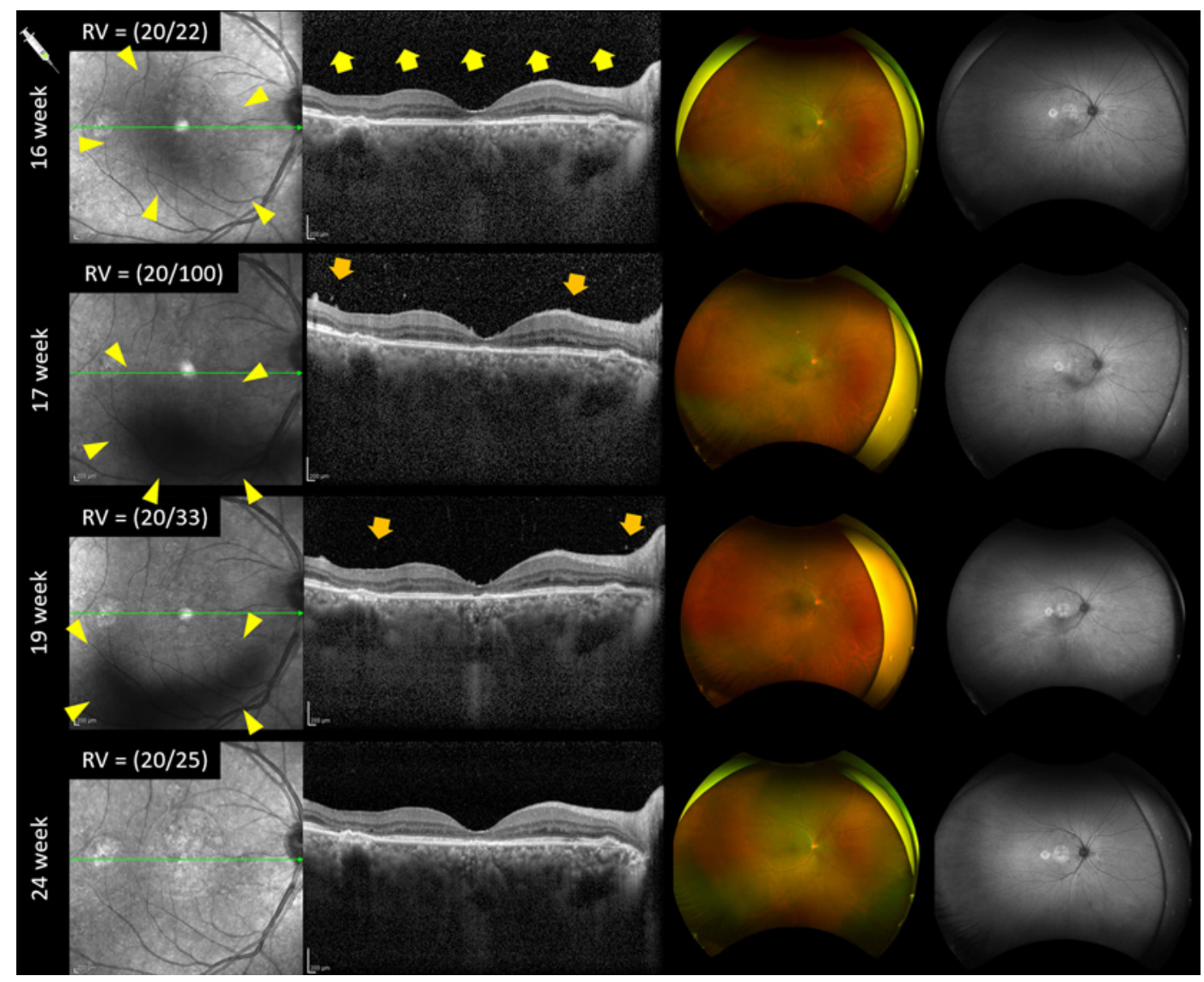

Fig. 3. After IVBr injection began, the first IVBr injection of a treat-and-extend regimen was administered at 16 weeks (Fig. 2). Five days later, the patient returned to the clinic complaining of decreased visual acuity after IVBr injection (17 weeks). At 17 weeks, her visual acuity had decreased to $20 / 100$, and fundus photograph and fundus autofluorescence showed vitreous opacities. The black smoke-like shadows (yellow arrowhead) seen in infrared images and vitreous noise (yellow arrow) seen in OCT images became thicker. Moreover, vitreous cells (orange arrow) were also seen on OCT images. The patient was then diagnosed with brolucizumab-related intraocular inflammation. The patient was immediately treated with 6 rounds of betamethasone drops, $40 \mathrm{mg}$ oral prednisolone, and $40 \mathrm{mg}$ subtenon triamcinolone acetonide injection. After starting anti-inflammatory treatment, the intraocular inflammation gradually subsided, and the black smoke-like shadows on infrared images and vitreous noise on OCT images also decreased (19 and 24 weeks). IVBr, intravitreal brolucizumab; OCT, optical coherence tomography.

IVBr injection may have accelerated the inflammation. However, since this obvious shadow and noise appeared at 16 weeks after IVBr injection and disappeared with anti-inflammatory treatment, our findings suggest that vitreous noise on OCT images can be considered to represent early-stage IOI. When following patients receiving IVBr treatment, OCT may be able to detect IOI at an earlier stage by evaluating vitreous haze.

There are several limitations in this study. This is a single case report, and it is necessary to collect more cases and evaluate them quantitatively in the future. In addition, since OCT noise is affected by different scanning algorithms, it is necessary to examine the optimal scan method, such as averaging or the use of swept-source OCT. Moreover, although the detection of anterior chamber inflammation by slit-lamp examination was subjective in this study, it also needs to be quantitatively assessed using laser flare photometry [15]. Finally, it is very difficult to distinguish whether this IOI is infectious 
endophthalmitis or noninfectious endophthalmitis related to IVBr. In this case, because of the lack of ocular discharge, the absence of pain, the absence of hypopyon, and the positive response to steroid treatment, we confirmed that it was noninfectious IOI. However, we cannot completely rule out bacterial endophthalmitis because we did not perform culture or polymerase chain reaction examinations.

Our retrospective review of the previous OCT suggests 2 clinically relevant points. First, IVBr may induce inflammation or may aggravate undetected negligible inflammation. Second, when a "shadow" is found on OCT, assessing the signal decay of the corresponding retinal image may be useful to determine its cause as vitreous inflammation or just random noise. In conclusion, new image noise and vitreous haze might be objective findings that suggest early IOI.

\section{Acknowledgment}

The authors thank Editage (https://www.editage.jp) for the English language review.

\section{Statement of Ethics}

This study was approved by the ethics committee of Saitama Medical University on June 9,2021 . Written informed consent was obtained from the patient in this case report and for publication of this article and the accompanying images. A copy of the written consent is available for review by the editor of this journal.

\section{Conflict of Interest Statement}

The authors have no conflicts of interest to declare.

\section{Funding Sources}

This study was supported in part by the Japan Society for the Promotion of Science (JSPS; KAKENHI Grant No. 21K16904).

\section{Author Contributions}

T.K. cared for the patient. Y.Y. determined the treatment protocol, prepared the figures, collected data, analyzed the ophthalmological findings, and gave critical suggestions. Y.Y. and K.S. prepared the manuscript.

\section{Data Availability Statement}

All data generated or analyzed during this study are included in this article and/or its online suppl. material files. Further enquiries can be directed to the corresponding author.

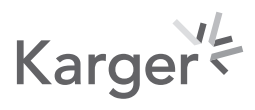


Yoshikawa et al.: Vitreous Noise on OCT and Brolucizimab-Related IOI

\section{References}

1 CATT Research Group; Martin DF, Martin DF, Maguire MG, Ying GS, Grunwald JE, Fine SL, et al. Ranibizumab and bevacizumab for neovascular age-related macular degeneration. N Engl J Med. 2011 May;364(20):1897908.

2 Heier JS, Brown DM, Chong V, Korobelnik JF, Kaiser PK, Nguyen QD, et al. Intravitreal aflibercept (VEGF trapeye) in wet age-related macular degeneration. Ophthalmology. 2012 Dec;119(12):2537-48.

3 Gillies MC, Hunyor AP, Arnold JJ, Guymer RH, Wolf S, Ng P, et al. Effect of ranibizumab and aflibercept on bestcorrected visual acuity in treat-and-extend for neovascular age-related macular degeneration: a randomized clinical trial. JAMA Ophthalmol. 2019 Apr;137(4):372-9.

4 Rofagha S, Bhisitkul RB, Boyer DS, Sadda SR, Zhang K, SEVEN-UP Study Group. Seven-year outcomes in ranibizumab-treated patients in ANCHOR, MARINA, and HORIZON: a multicenter cohort study (SEVEN-UP). Ophthalmology. 2013 Nov;120(11):2292-9.

5 Kim LN, Mehta H, Barthelmes D, Nguyen V, Gillies MC. Metaanalysis of real-world outcomes of intravitreal ranibizumab for the treatment of neovascular age-related macular degeneration. Retina. 2016 Aug;36(8): 1418-31.

6 Koh A, Lai TYY, Takahashi K, Wong TY, Chen LJ, Ruamviboonsuk P, et al. Efficacy and safety of ranibizumab with or without verteporfin photodynamic therapy for polypoidal choroidal vasculopathy: a randomized clinical trial. JAMA Ophthalmol. 2017 Nov;135(11):1206-13.

7 Dugel PU, Jaffe GJ, Sallstig P, Warburton J, Weichselberger A, Wieland M, et al. Brolucizumab versus aflibercept in participants with neovascular age-related macular degeneration: a randomized trial. Ophthalmology. 2017 Sep;124(9):1296-304.

8 Dugel PU, Koh A, Ogura Y, Jaffe GJ, Schmidt-Erfurth U, Brown DM, et al. HAWK and HARRIER: phase 3, multicenter, randomized, double-masked trials of brolucizumab for neovascular age-related macular degeneration. Ophthalmology. 2020 Jan;127(1):72-84.

9 Mones J, Srivastava SK, Jaffe GJ, Tadayoni R, Albini TA, Kaiser PK, et al. Risk of inflammation, retinal vasculitis, and retinal occlusion-related events with brolucizumab: post hoc review of HAWK and HARRIER. Ophthalmology. 2020 Jul;128(7):1050-9.

10 Baumal CR, Bodaghi B, Singer M, Tanzer DJ, Seres A, Joshi MR, et al. Expert opinion on management of intraocular inflammation, retinal vasculitis, and vascular occlusion after brolucizumab treatment. Ophthalmol Retina. 2021 Jun;5(6):519-27.

11 Ray R, Stinnett SS, Jaffe GJ. Evaluation of image artifact produced by optical coherence tomography of retinal pathology. Am J Ophthalmol. 2005 Jan;139(1):18-29.

12 Wu W, Tan O, Pappuru RR, Duan H, Huang D. Assessment of frame-averaging algorithms in OCT image analysis. Ophthalmic Surg Lasers Imaging Retina. 2013 Mar-Apr;44(2):168-75.

13 Saito M, Barbazetto IA, Spaide RF. Intravitreal cellular infiltrate imaged as punctate spots by spectral-domain optical coherence tomography in eyes with posterior segment inflammatory disease. Retina. 2013 Mar;33(3): 559-65.

14 Keane PA, Karampelas M, Sim DA, Sadda SR, Tufail A, Sen HN, et al. Objective measurement of vitreous inflammation using optical coherence tomography. Ophthalmology. 2014 Sep;121(9):1706-14. al

15 Lages V, Gehrig B, Herbort CP Jr. Laser flare photometry: a cost-effective method for early detection of endophthalmitis after intravitreal injection of anti-VEGF agents. J Ophthalmic Inflamm Infect. 2018 Dec;8(1):23.

\section{Karger ${ }^{\prime}=$}

